\title{
ON THE STABILITY OF A STEADY CONVECTIVE FLOW IN A VERTICAL LAYER OF A CHEMICALLY REACTING FLUID
}

\author{
ARMANDS GRITSANS*, VALENTINA KOLISKINA ${ }^{\dagger}$, ANDREI \\ KOLYSHKIN $^{\dagger}$, AND FELIX SADYRBAEV* \\ *Department of Physics and Mathematics \\ Daugavpils University \\ 1 Parades street, Daugavpils, LV5401, Latvia \\ e-mail: armands.gricans@du.lv, felix@latnet.lv \\ ${ }^{\dagger}$ Department of Engineering Mathematics \\ Riga Technical University \\ 2 Daugavgrivas street, Riga, LV1007, Latvia \\ e-mail: valentina.koliskina@rtu.lv, andrejs.koliskins@rtu.lv
}

Key words: Nonlinear Heat Sources, Bifurcation Analysis, Boundary Value Problem, Linear Stability

\begin{abstract}
Linear stability of a steady flow of a chemically reacting fluid located in a vertical fluid layer bounded by two infinite parallel planes is investigated. Steady convective flow in the vertical direction is initiated due to the combined effect of internal heat generation and the temperature difference between the planes. Imposing small perturbations on the base flow, linearizing equations of thermal convection under the Boussinesq approximation in the neighbourhood of the base flow and using the method of normal modes we obtain an eigenvalue problem for a system of ordinary differential equations. Collocation method is used to discretize the problem. Numerical calculations are performed in Matlab. Fluid velocity, pressure, and temperature are the solutions of a nonlinear boundary value problem. Properties of the nonlinear boundary value problem for the base flow are investigated numerically using bifurcation analysis. It is shown that both the temperature difference between the planes and intensity of internal heat generation have a destabilizing influence on the base flow. The intensity of heat transfer in the direction perpendicular to the main flow can promote instability and leads to more intensive mixing. This fact can be used in design of bioreactors for biomass thermal conversion.
\end{abstract}




\section{INTRODUCTION}

Development of our society in the near future will be dependent on the availability of alternative sources of energy. One promising area of investigation is biomass thermal conversion $[1,2]$. Researchers try to understand experimentally the importance of different factors (such as applied electrical field, intensity of chemical reactions, fluid mixing) on the efficiency of the conversion process $[3,4]$. Alternative approach is based on numerical modeling [5]. Biomass thermal conversion is described by complex interaction of the following processes: combustion, heat transfer, and fluid flow. One popular method of investigation of complex processes in fluid mechanics is stability analysis [6, 7]. Stability of convective flows is a topic of continuous interest due to numerous applications in physics and engineering. In some cases (biomass thermal conversion is one example) instability is desired since it results in more intensive mixing and (possibly) in more efficient energy conversion. Linear stability analysis of a steady convective flow due to internal heat generation in a vertical fluid layer is analyzed in [7]. Stability of a stationary flow in an annulus in the presence of a nonlinear heat source is analyzed in [8]. In the present paper we consider a linear stability problem that takes into account heat generated in the fluid as a result of chemical reaction and the tempetature difference across the fluid layer.

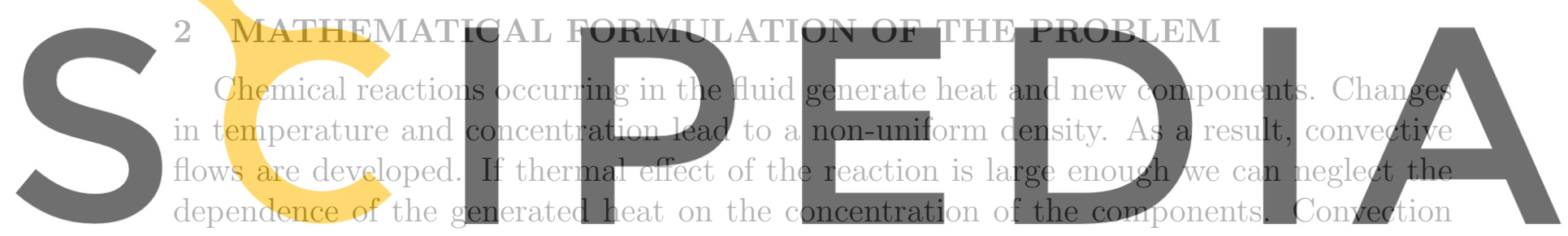

is then generated due to internal heat sources distributed within the fluid in accordance

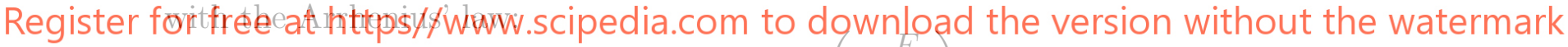

$$
Q=Q_{0} \exp \left(-\frac{E}{R T}\right)
$$

where $T$ is the temperature, $R$ is the universal gas constant and $E$ is the parameter characterizing reaction.

Consider an infinitely long vertical fluid layer filled with viscous incompressible fluid. The walls $\tilde{x}= \pm h$ are maintained at constant temperatures $\hat{T}$ and $-\hat{T}$, respectively. We let the measures of length, time, velocity, temperature and pressure be $h, h^{2} / \nu$, $\hat{g} \hat{\beta} h^{2} \hat{T} /(\nu E), R \hat{T}^{2} / E, \rho \hat{g} \hat{\beta} h R \hat{T}^{2} / E$, respectively. Here $\rho$ is the density, $\hat{\beta}$ is the coefficient of thermal expansion, $\hat{g}$ is the acceleration due to gravity and $\nu$ is the kinematic viscosity. Let $(x, y, z)$ be a system of Cartesian coordinates whose origin lies in the middle of the two planes $\tilde{x}= \pm h$ and the $z$-axis ir directed upwards. The flow is described by the 
system of equations of thermal convection under the Boussinesq approximation:

$$
\begin{aligned}
\frac{\partial \mathbf{v}}{\partial t}+G r(\mathbf{v} \nabla) \mathbf{v} & =\nabla p+\Delta \mathbf{v}+T \mathbf{k} \\
\frac{\partial T}{\partial t}+G r \mathbf{v} \nabla T & =\frac{1}{P r} \Delta T+\frac{F}{P r} \exp T \\
\nabla \mathbf{v} & =0
\end{aligned}
$$

where $\mathbf{v}$ and $p$ are the velocity and pressure, respectively, and $\mathbf{k}$ is the unit vector in the positive $z$-direction. The flow is characterized by three dimensionless parameters: the Grashof number $G r=\hat{g} \hat{\beta} R \hat{T}^{2} h^{3} /\left(\nu^{2} E\right)$, the Frank-Kamenetskii parameter $F=$ $Q_{0} E h^{2} /\left(\kappa R \hat{T}^{2}\right) \exp (-E /(R \hat{T}))$ and the Prandtl number $\operatorname{Pr}=\nu / \chi$, where $\chi=\kappa /\left(\rho c_{p}\right)$, $\kappa$ is the thermal conductivity, $c_{p}$ is the specific heat. The Frank-Kamenetskii transformation is used to represent the source term in (3). Thus, there are two factors that induce convection: (a) internal heat sources (1) and temperature gradient across the layer due to difference in the temperatures of the walls.

These two factors generate a steady convective flow in the vertical direction. Equations (2)-(4) have a steady solution of the following form

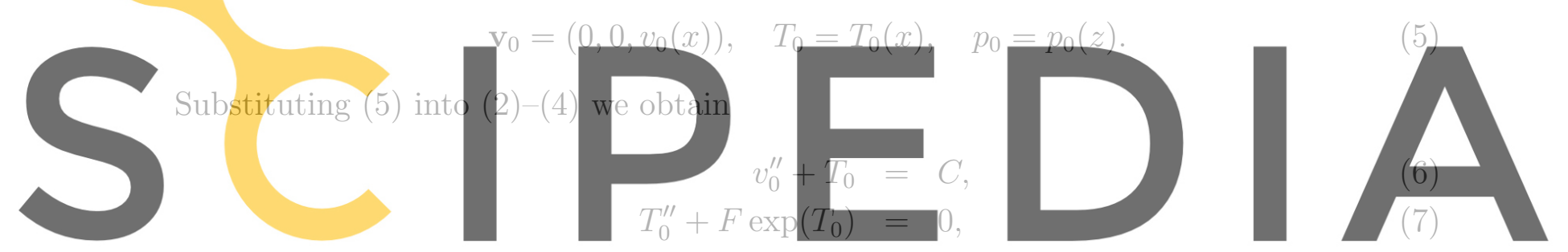

Register for free at https//www.scipedia.com to download the version without the watermark

$$
\begin{aligned}
& v_{0}( \pm 1)=0, \\
& T_{0}(\mp 1)= \pm 1 .
\end{aligned}
$$

The layer is assumed to be closed so that the total fluid flux through the cross-section is equal to zero:

$$
\int_{-1}^{1} v_{0}(x) d x=0
$$

It is seen from (6)-(10) that the problem for the temperature distribution $T_{0}(x)$ can be solved independently. In the next section we consider solution of the boundary value problem for $T_{0}(x)$.

\section{SOLUTION OF THE NONLINEAR BOUNDARY VALUE PROBLEM}

Let $F$ be a positive number. Consider the boundary value problem (7), (9). Let $T_{0}(x)$ be a solution of $(7)$, (9). Then, $T_{0}^{\prime \prime}(x)=-F e^{T_{0}(x)}<0$ for every $x \in[-1,1]$ and thus $T_{0}(x)$ is a strictly concave function in the interval $[-1,1]$ and $T_{0}^{\prime}(1)<0$. 
Let us consider the initial value problem (7),

$$
T_{0}(1)=-1, \quad T_{0}^{\prime}(1)=-\beta<0 .
$$

It follows from (7), (11) that

$$
T_{0}^{\prime 2}(x)=\beta^{2}+2 F e^{-1}-2 F e^{T_{0}(x)} .
$$

Integrating (12), we have

$$
\int_{-1}^{T_{0}(x)} \frac{d u}{ \pm \sqrt{\beta^{2}+2 F e^{-1}-2 F e^{u}}}=x-1,
$$

where "+" sign is valid on intervals, where $T_{0}(x)$ increases and, consequently, "-" sign is for intervals, where $T_{0}(x)$ decreases.

Using formula 1.3.1.8 on p. 137 in [10], we obtain from (13) that

$$
T_{0}(x)=\ln \left(\frac{4\left(\beta^{2}+2 F e^{-1}\right) e^{-1+(x-1) \sqrt{\beta^{2}+2 F e^{-1}}}}{\left[\beta\left(-1+e^{(x-1) \sqrt{\beta^{2}+2 F e^{-1}}}\right)+\sqrt{\beta^{2}+2 F e^{-1}}\left(1+e^{(x-1) \sqrt{\beta^{2}+2 F e^{-1}}}\right)\right]^{2}}\right)
$$
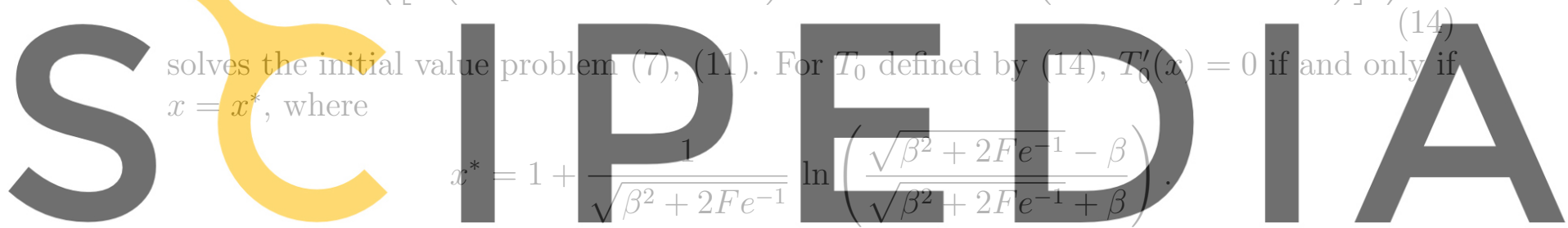

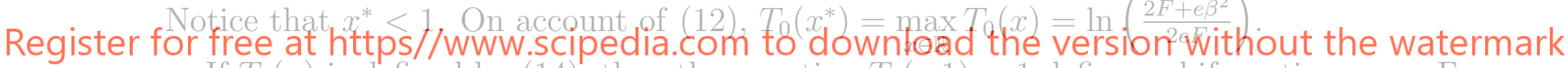
If $T_{0}(x)$ is defined by $(14)$, then the equation $T_{0}(-1)=1$ defines a bifurcation curve I

in the positive quadrant of the $(F, \beta)$-plane which determine all solutions of the boundary value problem (7), (9). By calculations, $T_{0}(-1)=1$ if and only if $G(F, \beta)=0$, where

$$
\begin{aligned}
G(F, \beta):=4 e^{2\left(-1+\sqrt{\beta^{2}+2 F e^{-1}}\right)}\left(\beta^{2}+2 F e^{-1}\right)- & \\
- & {\left[\left(1-e^{2 \sqrt{\beta^{2}+2 F e^{-1}}}\right) \beta+\left(1+e^{2 \sqrt{\beta^{2}+2 F e^{-1}}}\right) \sqrt{\beta^{2}+2 F e^{-1}}\right]^{2}, }
\end{aligned}
$$

and thus $\Gamma=\left\{(F, \beta) \in \mathbb{R}^{2}: F>0, \beta>0, G(F, \beta)=0\right\}$; the curve $\Gamma$ is depicted in Figure 1. Notice that $G(0,1)=0$. The curve $\Gamma$ has a turning point $A_{0}=\left(F_{0}, \beta_{0}\right)=$ $(0.7887,2.5363)$ (the brown point in Figure 1$)$ from right to left. We conclude that the boundary value problem (7), (9) has exactly two solutions if $F \in\left(0, F_{0}\right)$, exactly one solution if $F=F_{0}$, and has no solutions if $F>F_{0}$.

Let us introduce the region $D:=\left\{(F, \beta) \in \mathbb{R}^{2}: F>0, \beta>0, x^{*}>-1\right\}$ and the curve $\Lambda:=\left\{(F, \beta) \in \mathbb{R}^{2}: F>0, \beta>0, x^{*}=-1\right\}$; see the shaded region and the black curve, respectively, in Figure 1. We mention some properties of solutions to (7), (9). 
1. For the points $(F, \beta) \in \Gamma$ that are located in $D$, the corresponding solutions $T_{0}$ of $(7),(9)$ have a maximum in the interval $[-1,1]$, which is greater than one, and thus $T_{0}^{\prime}(-1)>0$; see Figure 2 and the blue solutions depicted in Figure 3, 4.

2. The curves $\Gamma$ and $\Lambda$ have a unique intersection point

$$
A_{2}=\left(\frac{1}{8 e} \ln ^{2}\left(2 e \sqrt{e^{2}-1}+2 e^{2}-1\right), \frac{\sqrt{e^{2}-1}}{2 e} \ln \left(2 e \sqrt{e^{2}-1}+2 e^{2}-1\right)\right)
$$

see Figure 1. The solution $T_{0}$ of (7), (9) that corresponds to the point $A_{2}$ is strictly decreasing in the interval $[-1,1]$ and $T_{0}^{\prime}(-1)=0$; see the green solution in Figure 3 .

3. For the points $(F, \beta) \in \Gamma$ that are located outside of $D$ and that are different from $A_{2}$. the corresponding solutions $T_{0}$ of $(7),(9)$ are strictly decreasing in the interval $[-1,1]$ and $T_{0}^{\prime}(-1)<0$; see the green solution in Figure 4. We note that for such solutions $T_{0}$ the formula (12) should be used with "-" sign and $\int_{-1}^{1} \frac{d u}{\sqrt{\beta^{2}+2 F e^{-1}-2 F e^{u}}}=2$.
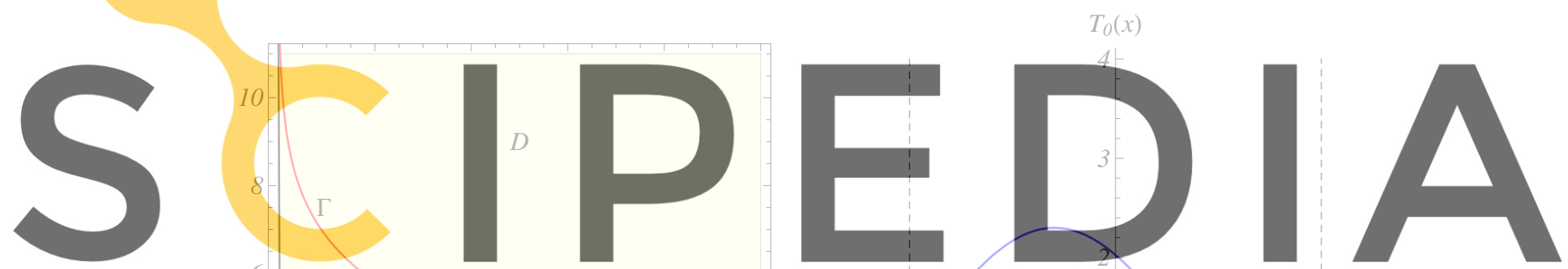

Register for free at https//Www
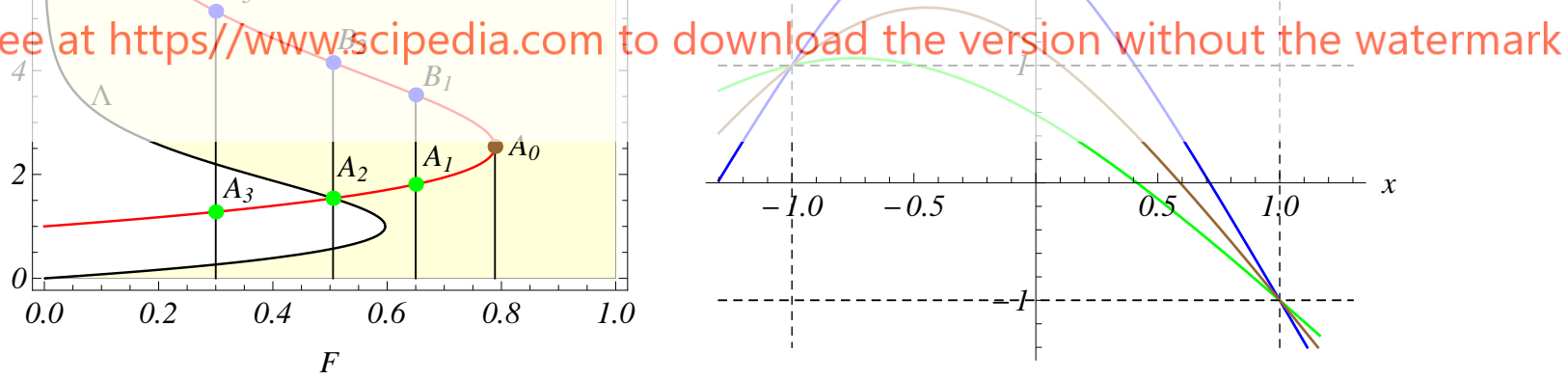

Figure 2: Three solutions of (7), (9) corresponding to the points $A_{1}=(0.65,1.8102) \in \Gamma, B_{1}=$ $(0.65,3.5329) \in \Gamma$, and $A_{0}=(0.7887,2.5363) \in \Gamma$

Figure 1: The bifurcation curve $\Gamma$ (red). depicted in Figure 1.

The results presented in this section allow one to select a physically realizable solution (corresponding to a point that is located on the lower branch of $\Gamma$ ), which is used later in linear stability analysis. 


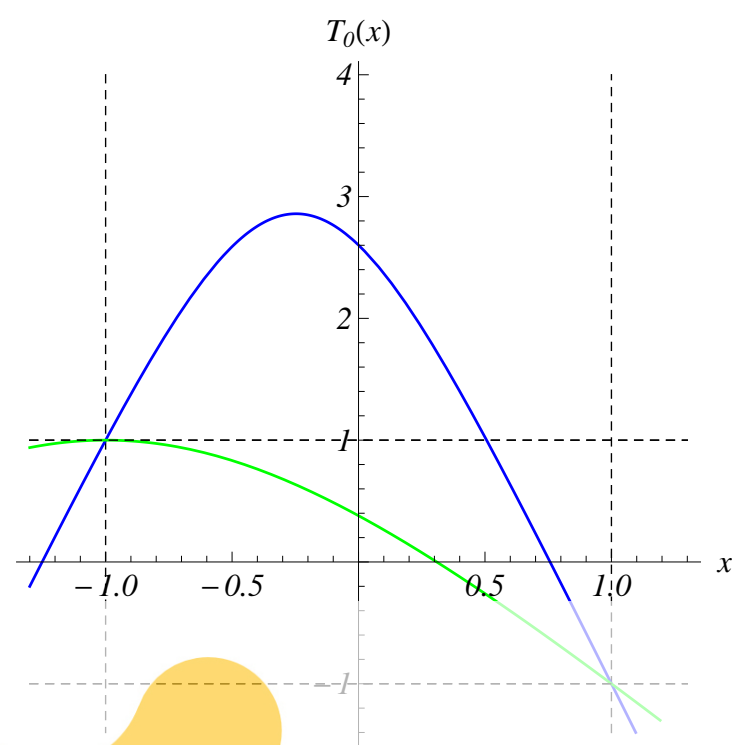

Figure 3: Two solutions of (7), (9) corresponding to the points $A_{2}=(0.5053,1.5412) \in \Gamma$ and $B_{2}=(0.5053,4.1538) \in \Gamma$ depicted in Figure 1.

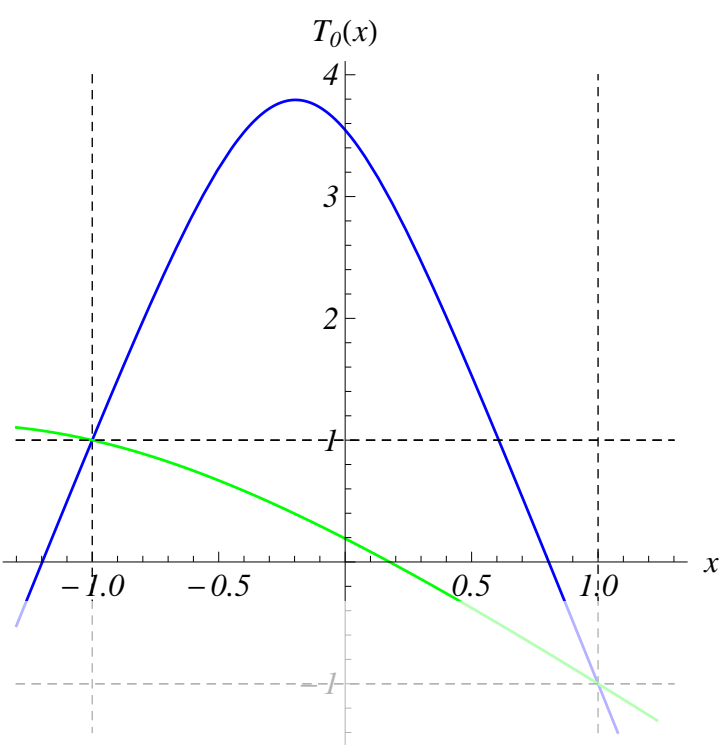

Figure 4: Two solutions of (7), (9) corresponding to the points $A_{3}=(0.3,1.2791) \in \Gamma$ and $B_{3}=(0.3,5.1406) \in \Gamma$ depicted in Figure 1.
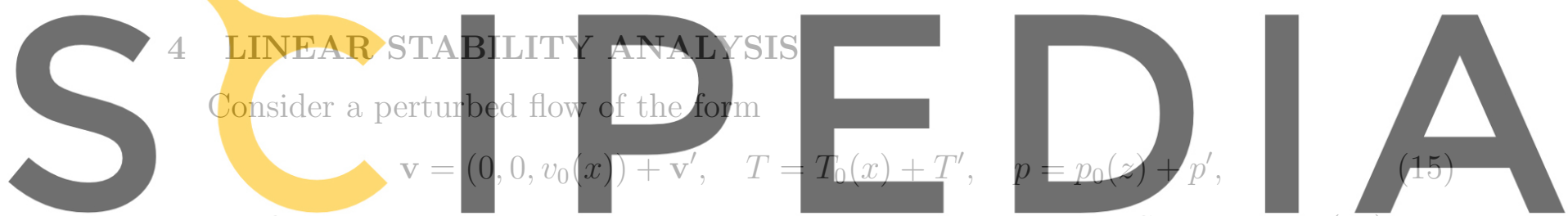

where flow quantities with primes represent small perturbations. Substituting (15) into

Register for free at hattps $/ \mathrm{s}$ ww w. ducing the normal modes

$$
\psi^{\prime}=\varphi(x) e^{-\lambda t+i k z}, \quad T^{\prime}=\vartheta(x) e^{-\lambda t+i k z},
$$

where $\psi^{\prime}$ is the stream function for the perturbed velocity field and $\lambda=\lambda_{r}+i \lambda_{i}$ is a complex eigenvalue, we obtain the eigenvalue problem

$$
\begin{gathered}
\varphi^{(4)}-2 k^{2} \varphi^{\prime \prime}+k^{4} \varphi+i k G r\left(\varphi v_{0}^{\prime \prime}-v_{0} \varphi^{\prime \prime}+k^{2} v_{0} \varphi\right)+\theta^{\prime}=-\lambda\left(\varphi^{\prime \prime}-k^{2} \varphi\right), \\
\frac{1}{P r}\left(\theta^{\prime \prime}-k^{2} \theta\right)+\frac{F}{P r} e^{T_{0}} \theta+i k G r\left(\varphi T_{0}^{\prime}-v_{0} \theta\right)=-\lambda \theta, \\
\varphi( \pm 1)=0, \quad \varphi^{\prime}( \pm 1)=0, \quad \theta( \pm 1)=0 .
\end{gathered}
$$

Problem (17)-(19) is solved by collocation method. The functions $\varphi(x)$ and $\theta(x)$ are approximated as follows

$$
\varphi(x)=\sum_{n=0}^{N} a_{n}\left(1-x^{2}\right)^{2} T_{n}(x), \quad \theta(x)=\sum_{n=0}^{N} b_{n}\left(1-x^{2}\right) T_{n}(x),
$$




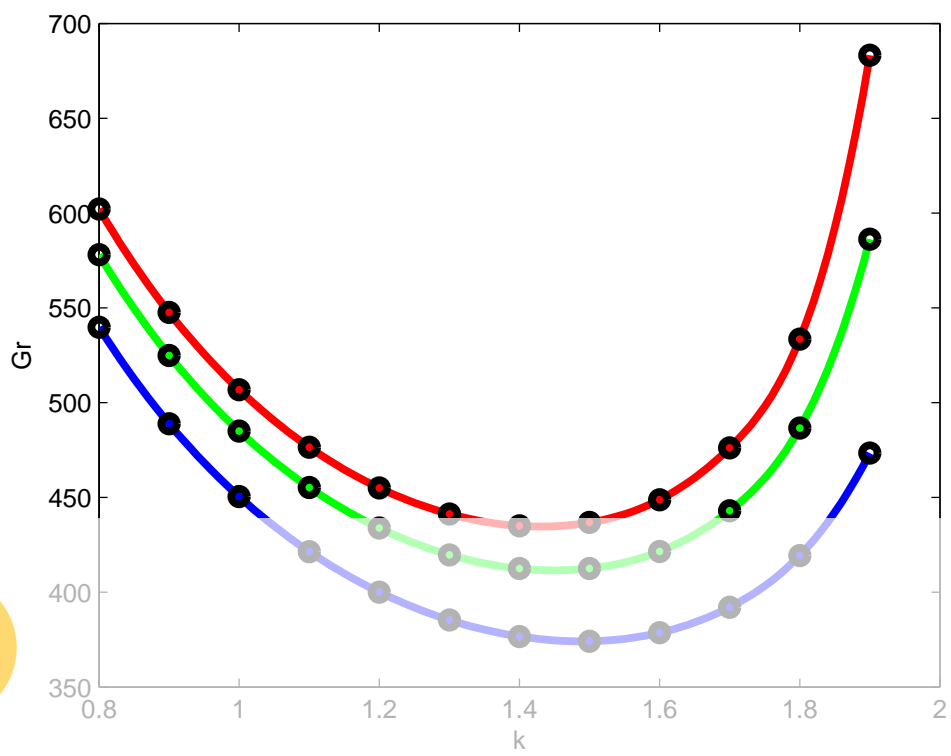

Figure 5: Marginal stability curves for $F=0.3$ (red curve), $F=0.5$ (green curve) and $F=0.7$ (blue
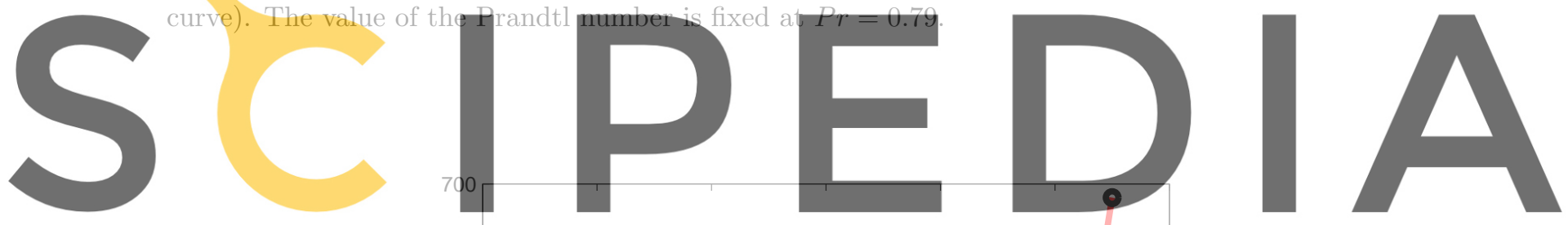

Register for free at https//www.scipedia.com to download the version without the watermark

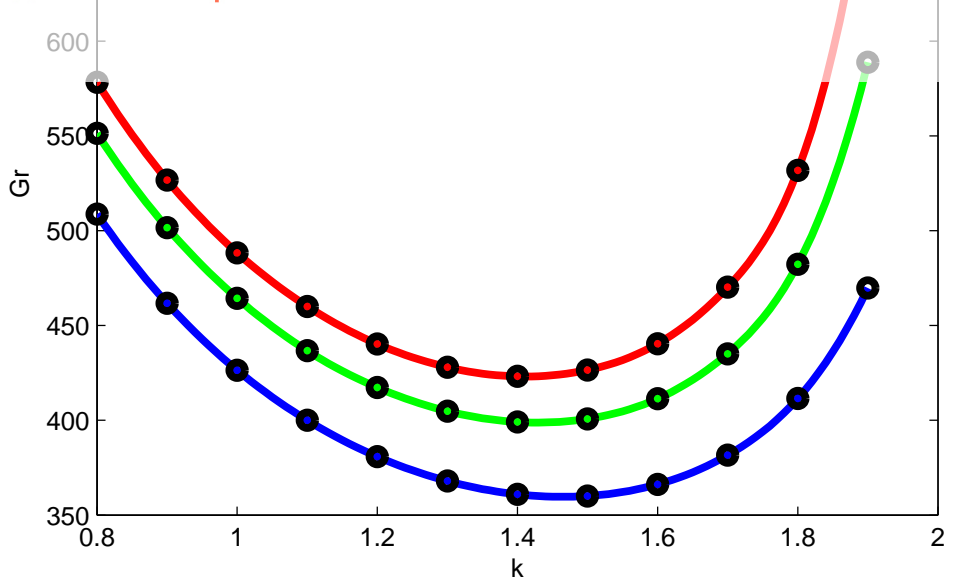

Figure 6: Marginal stability curves for $F=0.3$ (red curve), $F=0.5$ (green curve) and $F=0.7$ (blue curve). The value of the Prandtl number is fixed at $\operatorname{Pr}=3$. 
where $T_{n}(x)$ is the Chebyshev polynomial of the first kind of order $n$. The collocation points are

$$
x_{j}=\cos \frac{\pi j}{N}, \quad j=0,1, \ldots, n
$$
form

Using (20), (21) we transform (17)-(19) to the generalized eigenvalue problem of the

$$
(A+\lambda B) \mathbf{a}=0
$$

where $\mathbf{a}=\left(a_{0} a_{1} \ldots a_{n} b_{0} b_{1} \ldots b_{n}\right)^{T}$. Problem (22) is solved numerically in Matlab for different values of the parameters of the problem.

Marginal stability curves for the case $\operatorname{Pr}=0.79$ are shown in Fig. 5 for three values of the parameter $F$. Black points on each graph represent calculated values.

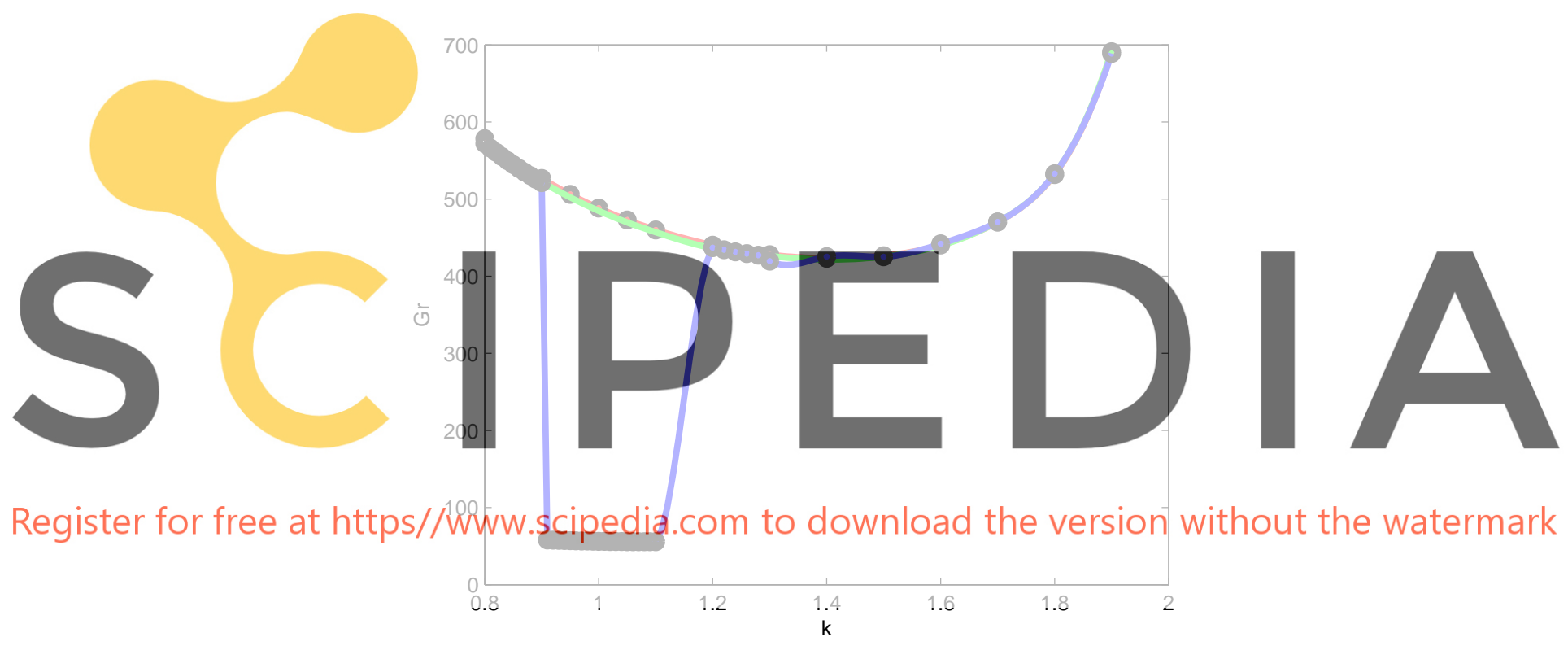

Figure 7: Marginal stability curves for $\operatorname{Pr}=3$ (red curve), $\operatorname{Pr}=10$ (green curve) and $\operatorname{Pr}=100$ (blue curve).

Calculated marginal stability curves for the case $\mathrm{Pr}=3$ are shown in Fig. 6. Comparing Figs. 5 and 6 we see that there are two destabilizing factors: the flow becomes less stable if the parameters $F$ (the intensity of the chemical reaction) and $\operatorname{Pr}$ increase.

Another set of calculations is performed for the case $F=0.3$ and three different Prandtl numbers. The results are plotted in Fig. 7. It is seen that for moderate range of the Prandtl numbers $(3<\operatorname{Pr}<10)$ the stability boundary is insensitive to the change in $\operatorname{Pr}$ (the red and green curves almost coincide in Fig. 7). For large Prandtl numbers $(\operatorname{Pr}=100)$ the critical Grashof number decreases considerably. In addition, the most 


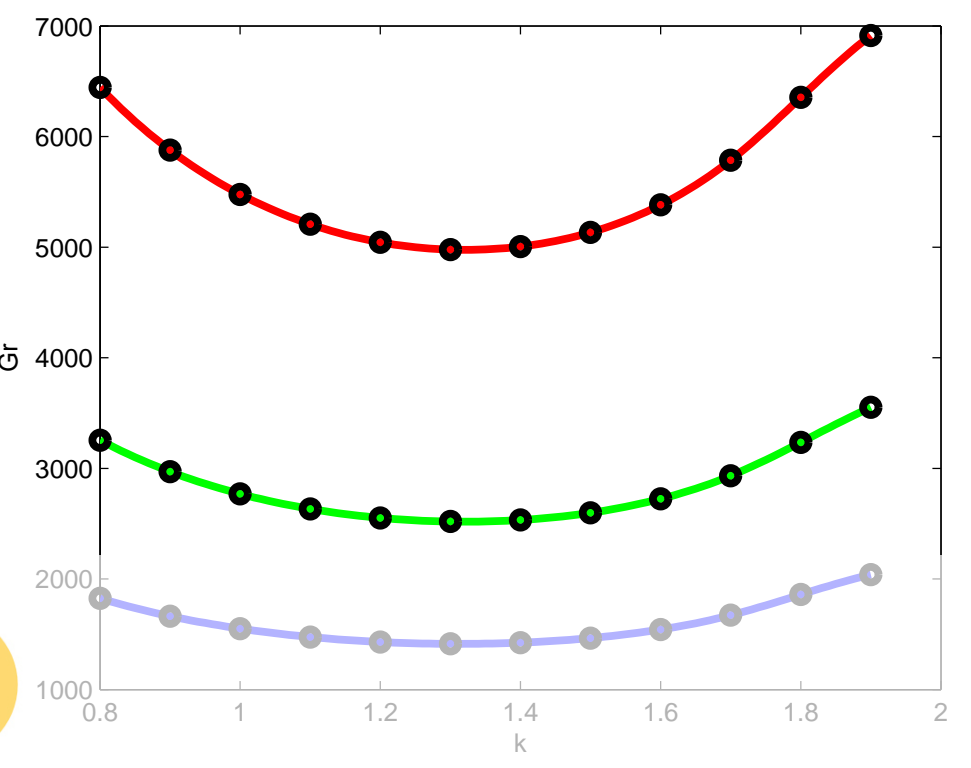

Figure 8: Marginal stability curves for $F=0.3$ (red curve), $F=0.5$ (green curve) and $F=0.7$ (blue curve). The value of the Prandtl number is fixed at $\operatorname{Pr}=0.79$.

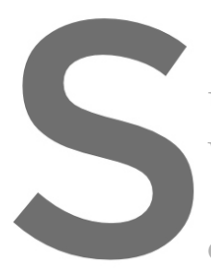
unstable mode in this case corresponds to perturbations with larger wave length (sma/ler
wave numbers).
The graphs in Figs. $5-7$ correspond to the case where there are ty factors inducing
convective flow: (a) internal heat generation and (b) constant dimensional temperature difference $2 \hat{T}$ between the walls. Let us compare our results with the case where only

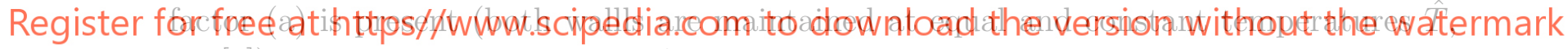
see [8]). Marginal stability curves for the case $P r=0.79$ with equal wall temperatures are shown in Fig. 8. Comparing Figs. 5 and 8 we see that the presence of the temperature gradient between the walls considerably destabilizes the flow. In addition, the minimum of the marginal stability curve is shifted to the left in the $(k, G r)$-plane. This means that perturbations of longer wavelength become more unstable as the Prandtl number grows.

\section{CONCLUSIONS}

Linear stability analysis of a steady flow of a chemically reacting fluid in a vertical layer bounded by two planes maintained at different constant temperatures is performed in the paper. Base flow solution is described by a nonlinear boundary value problem. The properties of this problem are investigated using bifurcation analysis. Results of linear stability calculations show that there are several destabilizing factors that affect linear stability: (a) the Prandtl number, (b) the Frank-Kamenetskii parameter. In addition, the presence of horizontal temperature gradient leads to considerable destabilization of the base flow in comparison with the case of equal temperatures of the walls. 


\section{ACKNOWLEDGMENT}

This work was supported by the Latvian Council of Science, project No. lzp-2020/10076 "Analysis of complex dynamical systems in fluid mechanics and heat transfer".

\section{REFERENCES}

[1] Yamakawa, C.K., Qin, F. and Mussatto, S.I. Advances and opportunities in biomass conversion technologies and biorefineries for the development of bio-based economy. Biomass and Bioenergy (2018) 119:54-60.

[2] Lewandowski, W.M., Ryms, M. and Kosakowski, W. Thermal biomass conversion: a review. Processes (2020) 8:doi:10.3390/pr8050516.

[3] Barmina, I., Purmalis, M., Valdmanis, R. and Zake M. Electrodynamic control of the combustion characteristics and heat energy production. Combustion Science and Technology (2016) 188:190-206.

\section{[4] Barmina, I., Valdmanis, R. and Zake M. The effect of biomass co-gasification and} co-firing on the development of combustion dynamics. Energy (2018) 146:4-12.
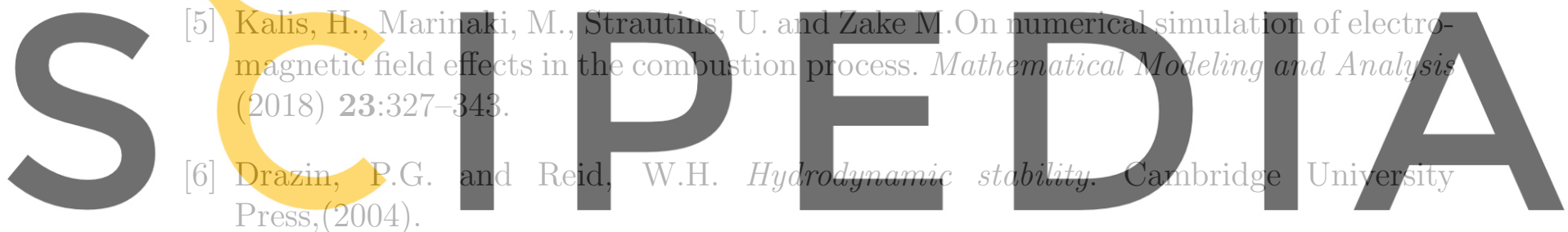

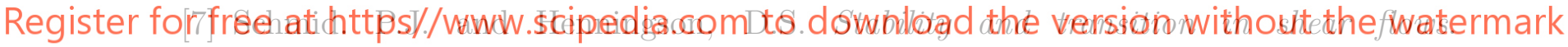
Springer, (2001).

[8] Eremin E.A. Stability of the stationary plane-parallel convective motion of a chemically active medium. Fluid Dynamics (1983) 18:438-441.

[9] Iltins, I., Iltina, M., Kolyshkin, A. and Koliskina, V. Linear stability of a convective flow in an annulus with a nonlinear heat source. JP Journal of Heat and Mass Transfer (2019) 18:315-329.

[10] Prudnikov, A.P., Brychkov Yu. A. and Marichev O.I. Integrals and Series. CRC, Vol. I., (1998). 